\title{
AN ALGEBRAIC FRAMEWORK OF WEIGHTED DIRECTED GRAPHS
}

\author{
PHILIPPE LEROUX
}

Received 19 February 2003

\begin{abstract}
We show that an algebraic formulation of weighted directed graphs leads to introducing a $k$-vector space equipped with two coproducts $\Delta$ and $\tilde{\Delta}$ verifying the so-called coassociativity breaking equation $(\tilde{\Delta} \otimes \operatorname{id}) \Delta=(\operatorname{id} \otimes \Delta) \tilde{\Delta}$. Such a space is called an $L$-coalgebra. Explicit examples of such spaces are constructed and links between graph theory and coassociative coalgebras are given.
\end{abstract}

2000 Mathematics Subject Classification: 05C20, 16W30.

1. Introduction. On the one hand, motivated by periodicity phenomena in algebraic $K$-theory, Loday [12] introduced the notion of "noncommutative Lie algebra," called Leibniz algebra. Such algebras $D$ are described by a bracket $[-, z]$ verifying the Leibniz identity:

$$
[[x, y], z]=[[x, z], y]+[x,[y, z]]
$$

When the bracket is skew-symmetric, the Leibniz identity becomes the Jacobi identity and the Leibniz algebra turns out to be a Lie algebra. A way to construct such a Leibniz algebra is to start with an associative dialgebra, that is, a $k$ vector space $D$ equipped with two associative products $\vdash$ and $\dashv$ such that for all $x, y, z \in D$,
(1) $x \dashv(y \dashv z)=x \dashv(y \vdash z)$,
(2) $(x \vdash y) \dashv z=x \vdash(y \dashv z)$,
(3) $(x \vdash y) \vdash z=(x \dashv y) \vdash z$.

The associative dialgebra is then a Leibniz algebra by defining $[x, y]:=x \dashv$ $y-y \vdash x$ for all $x, y \in D$. The operad of associative dialgebras is Koszul dual to the operad of dendriform algebras, a dendriform algebra $E$ being a $k$-vector space equipped with two binary operations $\prec, \succ: E \otimes E \rightarrow E$, satisfying the following axioms:

(1) $(a \prec b) \prec c=a \prec(b \prec c)+a \prec(b \succ c)$,

(2) $(a \succ b) \prec c=a \succ(b \prec c)$,

(3) $(a \prec b) \succ c+(a \succ b) \succ c=a \succ(b \succ c)$. 
This notion dichotomizes the notion of associativity since the product $a * b=$ $a \prec b+a \succ b$, for all $a, b \in E$, is associative. By dualizing, we can easily define the notions of coassociative codialgebras and dendriform coalgebras. Coassociative codialgebras are then $k$-vector spaces equipped with two coassociative coproducts $\Delta_{\text {dias }}$ and $\tilde{\Delta}_{\text {dias }}$ verifying $\left(\tilde{\Delta}_{\text {dias }} \otimes\right.$ id $) \Delta_{\text {dias }}=\left(\right.$ id $\left.\otimes \Delta_{\text {dias }}\right) \tilde{\Delta}_{\text {dias }}$, and two other axioms easily obtained from the definition of a dialgebra. Similarly, a dendriform coalgebra is a $k$-vector space equipped with two coproducts, not necessarily coassociative, $\Delta_{\text {dend }}$ and $\tilde{\Delta}_{\text {dend }}$, still verifying the same equation $\left(\tilde{\Delta}_{\text {dend }} \otimes\right.$ id $) \Delta_{\text {dend }}=\left(\right.$ id $\left.\otimes \Delta_{\text {dend }}\right) \tilde{\Delta}_{\text {dend }}$.

On the other hand, motivated by classical random walks on directed graphs and more generally by weighted directed graphs, we introduce an algebraic framework based on a particular $k$-vector space equipped with two coproducts $\Delta$ and $\tilde{\Delta}$ verifying $(\tilde{\Delta} \otimes$ id $) \Delta=(\operatorname{id} \otimes \Delta) \tilde{\Delta}$ called in the sequel the coassociativity breaking equation. Such a space is called an $L$-coalgebra. In this setting, bidirected graphs are characterized by the equation $\Delta=\tau \tilde{\Delta}$, where $\tau$ is the switch map. This leads us to introduce a special cocommutator $\operatorname{ker}(\Delta-\tau \tilde{\Delta})$. By dualizing, we also obtain an algebraic framework of algebras equipped with two products $\vdash$ and $\dashv$ and the commutator becomes $[x, y]:=x \dashv y-y \vdash x$. Requiring that $[-, z]$ verifies the "Leibniz identity" implies studying particular coalgebras called coassociative codialgebras. Therefore, requiring an algebraic framework for weighted directed graph leads also to considering special algebras equipped with two products.

These particular $L$-coalgebras, the coassociative codialgebras, and dendriform codialgebras, in addition to those coming from weighted graphs theory, are exciting motivations to investigate further algebras (resp., coalgebras) equipped with two or more products (resp., coproducts). Indeed, to construct associative dialgebras and dendriform algebras, the way is to start with constructing their coversions and to consider the $k$-vector space of linear maps defined on such spaces and taking values into an associative algebra. The convolution products defined from these two coproducts will yield associative dialgebras and dendriform algebras.

This paper is the first of a series of 6 papers $[7,8,9,10,11]$ on the constructions of $L$-coalgebras via graph theory. We very briefly summarize the obtained results. In [9], we focus on unital algebras viewed as $L$-bialgebras and show the existence of differential associative dialgebra associated with each curvature, in the sense of Quillen [15], of a Leibniz-Ito derivative, that is, a linear map $\rho: A \rightarrow M$ such that for all $x, y \in A, \rho(x y)=x \rho(y)+\rho(x) y+\rho(x) \rho(y)$, with $A$ an algebra with unit $1, M$ an $A$-bimodule, and $\rho(1)=0$. Motivated by a work of Joni and Rota [5] on combinatorics, the $L$-coalgebra formalism is also applied in [7]. We prove that the combinatorics generated by a quantum random walk over $\mathbb{Z}$, called the Hadamard walk, can be recovered from periodic orbits of a classical chaotic system. There exists a bijection between these periodic orbits and periodic orbits of a particular directed graph whose associated $L$-coalgebra 
is such that $\tilde{\Delta}=\Delta$. In [10], we show that any weighted directed graph, through its associated (Markov) $L$-coalgebra, yields solutions of the Yang-Baxter equation and thus provides representations of the braid groups. In [11], we construct (Markov) coassociative codialgebras and show a relationship between these codialgebras and a class of well-known coassociative coalgebras by considering a tool from graph theory called the line extension. We also exhibit a tiling of directed graphs called the $\left(n^{2}, 1\right)$-De Bruijn graphs made with $n$ coassociative coalgebras. These constructions were our first examples of coassociative manifolds [8]. We also obtain examples of cubical trialgebra, a notion defined in [13], and more generally examples of hypercube $n$-algebra, that is, a $k$-vector space $V$ equipped with $n$ products verifying $\left(x \bullet_{i} y\right) \bullet_{j} z=x \bullet_{i}\left(y \bullet_{j} z\right)$, $x, y, z \in V, i, j=0, \ldots, n-1$, as well as associative products which split into several ones, that is, $x \star y=\sum_{i} x \star_{i} y$, for all $x, y \in A$, with $\star_{i}$ associative, for all $i=0, \ldots, n-1$. In [8], we construct $L$-Hopf algebras, coassociative codialgebras, coassociative cotrialgebras, see [13] for the definition, dendriform coalgebras, and Poisson algebras. All these constructions led us to the notion of coassociative manifold developed in [8].

We now introduce the first part of the work on $L$-coalgebras. We start this paper in Section 2 with recalling the definition of a weighted directed graph and with introducing axioms of $L$-coalgebras. We construct from any weighted directed graphs an $L$-coalgebra called a Markov $L$-coalgebra. Then, we enlarge the definition of a directed graph and construct for any $L$-coalgebra its weighted directed graph. As a coassociative coalgebra is a trivial $L$-coalgebra, we study some consequences of this association, one of them being the nonlocality of the coassociative coproduct over such a graph. We prove also that any coassociative coalgebra $(C, \Delta)$ equipped with a group-like element can be viewed as a nontrivial $L$-coalgebra $(C, \vec{d}, \overleftarrow{d})$. The case of $a$ is then investigated and the new coproducts $\vec{d}$ and $\overleftarrow{d}$ turn out to be Leibniz-Ito derivatives. Motivated by this construction, we construct, in Section $4, L$-coalgebras from any Markov $L$ coalgebras $(L, \Delta, \tilde{\Delta})$ whose new coproducts $\vec{d}$ and $\overleftarrow{d}$ become Leibniz-Ito derivatives if coproducts $\Delta$ and $\tilde{\Delta}$ are unital homomorphisms. We also set a comparison between usual graph theory and coassociative coalgebras. This work ends with the example of the quaternions algebra embedded into a Markov L-Hopf algebra (of degree 2) whose associated directed graph is the directed triangle.

2. Definitions and notation. We denote by $k$ the field $\mathbb{R}$ or $\mathbb{C}$ and consider only unital associative algebras. The Sweedler notation $\Delta a=\sum_{a} a_{(1)} \otimes a_{(2)}$ will be also used. The transposition map will be denoted by $\tau: V_{1} \otimes V_{2} \otimes \cdots \otimes V_{n} \rightarrow$ $V_{n} \otimes V_{1} \otimes \cdots \otimes V_{n-1}$ such that $\tau\left(x_{1} \otimes x_{2} \otimes \cdots \otimes x_{n}\right)=x_{n} \otimes x_{1} \otimes \cdots \otimes x_{n-1}$, where $V_{1}, V_{2}, \ldots, V_{n}$ are $n k$-vector spaces. We recall that a unital associative algebra is a $k$-vector space $(A, m, \eta)$ equipped with a product $m: A \otimes A \rightarrow A$ verifying $m(m \otimes \mathrm{id})=m($ id $\otimes m)$ (associativity) and a unit map $\eta: k \rightarrow A, \lambda \mapsto \lambda 1_{A}$. Dualizing the previous definition, we obtain a coassociative coalgebra over $k$ 
$[14,17]$, that is, a $k$-vector space $(C, \Delta, \epsilon)$ such that the counit map $\epsilon: C \rightarrow k$ and the coproduct map $\Delta: C \rightarrow C \otimes C$ verify

(1) the coassociativity equation $(\Delta \otimes$ id $) \Delta=($ id $\otimes \Delta) \Delta$;

(2) the counit equation (id $\otimes \epsilon) \Delta=\mathrm{id}=(\epsilon \otimes \mathrm{id}) \Delta$.

A coalgebra is said to be cocommutative if $\Delta=\tau \Delta$. Similarly, a bialgebra $(C, m, \eta, \Delta, \epsilon, k)$ over $k$ is a $k$-vector space such that $(C, \Delta, \epsilon)$ is a coalgebra, and $(C, m, \eta)$ is an algebra such that the coproduct and counit are algebra homomorphisms. A Hopf algebra $(H, m, \eta, \Delta, \epsilon, S, k)$ is a bialgebra with a $k$-linear map $S: H \rightarrow H$ called antipode which verifies $m(S \otimes \mathrm{id}) \Delta=m(\mathrm{id} \otimes S) \Delta=\eta \epsilon$. An antipode is unique, unital antialgebra map, and an anticoalgebra map, that is, for all $x \in H,(S \otimes S) \Delta x=\tau \Delta S(x)$.

DEFINITION 2.1 (directed graph). A directed graph $G$ is a quadruple ( $G_{0}, G_{1}$, $s, t$ ) [16], where $G_{0}$ and $G_{1}$ are two denumerable sets called, respectively, the vertex set and the arrow set. The two maps $s, t: G_{1} \rightarrow G_{0}$ are called, respectively, source and terminus. A vertex $v \in G_{0}$ is a source (resp., a sink) if $t^{-1}(\{v\})$ (resp., $\left.s^{-1}(\{v\})\right)$ is empty. A graph $G$ is said to be locally finite (resp., rowfinite) if $t^{-1}(\{v\})$ (resp., $\left.s^{-1}(\{v\})\right)$ is finite. Fix a vertex $v \in G_{0}$. Define the set $F_{v}:=$ $\left\{a \in G_{1}, s(a)=v\right\}$. A weight associated with the vertex $v$ is a map $w_{v}: F_{v} \rightarrow k$. A directed graph equipped with a family of weights $w:=\left(w_{v}\right)_{v \in G_{0}}$ is called a weighted directed graph.

REMARK 2.2. The case of nondirected graphs can be dealt with in this framework by imposing that for each arrow $a \in G_{1}$, such that $s(a)=u$ and $t(a)=v$, there exists a unique $\bar{a} \in G_{1}$ with $s(\bar{a})=v$ and $t(\bar{a})=u$. We then identify $a$ with $\bar{a}$. Should this identification be omitted, the graph is directed, the condition of existence of $\bar{a}$ meaning that every arrow has an inverse.

This subsection ends by recalling the definition of the line extension of a directed graph and the graph isomorphism.

Definition 2.3 (line extension). The line extension of a directed graph $G$ := $\left(G_{0}, G_{1}, s, t\right)$ with a denumerable vertex set $G_{0}$ and a denumerable arrow set $G_{1} \subseteq G_{0} \times G_{0}$ is the directed graph with vertex set $G_{1}$ and arrow set $G_{2} \subseteq G_{1} \times G_{1}$ defined by $(v, w) \in G_{1} \times G_{1}$ belongs to $G_{2}$ if and only if $t(v)=s(w)$. This directed graph, called the line-directed graph of $G$, is denoted by $E(G)$.

DEFINITION 2.4 (graph isomorphism). A graph isomorphism $f: G \rightarrow H$ between two graphs $G=\left(G_{0}, G_{1}, s_{G}, t_{G}\right)$ and $H=\left(H_{0}, H_{1}, s_{H}, t_{H}\right)$ is a pair of bijections $f_{0}: G_{0} \rightarrow H_{0}$ and $f_{1}: G_{1} \rightarrow H_{1}$ such that $f_{0}\left(s_{G}(a)\right)=s_{H}\left(f_{1}(a)\right)$ and $f_{0}\left(t_{G}(a)\right)=t_{H}\left(f_{1}(a)\right)$ for all $a \in G_{1}$. All the directed graphs in this formalism will be considered up to a graph isomorphism.

EXAMPLE 2.5. The two directed graphs in Figure 2.1 are isomorphic.

3. Coassociativity breaking. We introduce $L$-coalgebras and show why this notion is interesting. 

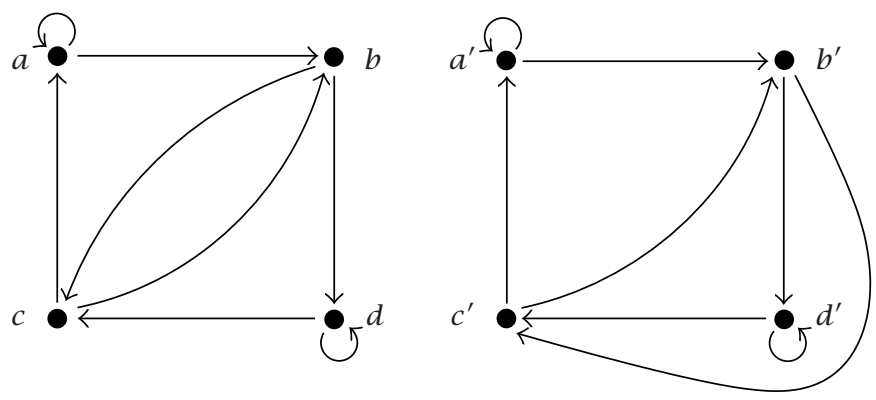

FIGURE 2.1

\subsection{Axioms}

DEFINITION 3.1 ( $L$-coalgebra). An $L$-coalgebra $(L, \Delta, \tilde{\Delta})$ is a $k$-vector space equipped with a right coproduct $\Delta: L \rightarrow L^{\otimes 2}$ and a left coproduct $\tilde{\Delta}: L \rightarrow L^{\otimes 2}$, verifying the coassociativity breaking equation $(\tilde{\Delta} \otimes \mathrm{id}) \Delta=(\mathrm{id} \otimes \Delta) \tilde{\Delta}$. An $L$ coalgebra may have two counits. The right counit $\epsilon: L \rightarrow k$ verifying (id $\otimes \epsilon$ ) $\Delta=$ id and the left counit $\tilde{\epsilon}: L \rightarrow k$ verifying $(\tilde{\epsilon} \otimes$ id $) \tilde{\Delta}=$ id. An $L$-coalgebra is called coassociative if its two coproducts are coassociative. In this case, the equation $(\tilde{\Delta} \otimes \mathrm{id}) \Delta=(\mathrm{id} \otimes \Delta) \tilde{\Delta}$ is called the entanglement equation, see $[8,11]$.

Proposition 3.2. Any coassociative coalgebra is an L-coalgebra.

Proof. Let $C$ be a coassociative coalgebra and $\Delta$ its coproduct. Set $\tilde{\Delta}:=\Delta$. The two coproducts verify $(\tilde{\Delta} \otimes$ id $) \Delta=($ id $\otimes \Delta) \tilde{\Delta}$.

The case $\tilde{\Delta}:=\Delta$ will be called the degenerate case. To discriminate between the different types of $L$-coalgebras, we give the following definition.

DEFINITION 3.3 (finite Markov $L$-coalgebra). A Markov L-coalgebra is an $L$ coalgebra $\left(\mathscr{G}, \Delta_{M}, \tilde{\Delta}_{M}\right)$, which is of dimension $\operatorname{dim} \mathscr{G}$ as a $k$-vector space, with a basis $\mathscr{G}_{0}:=\left(v_{i}\right)_{1 \leq i \leq \operatorname{dim} \varphi}$ equipped with

(1) a set $\mathscr{G}_{1}:=\left\{v_{i} \otimes v_{j} ;\left(v_{i}, v_{j}\right) \in \mathscr{G}_{0} \times \mathscr{G}_{0}\right\}$,

(2) two subsets $I_{v_{i}}$ and $J v_{v_{i}}$ of $\mathscr{G}_{1}$, and maps $w_{v_{i}}: I_{v_{i}} \rightarrow k$ and $\tilde{w}_{v_{i}}: J_{v_{i}} \rightarrow k$ called weights, for any $1 \leq i \leq \operatorname{dim} \mathscr{G}$, verifying that

$$
\begin{aligned}
& \Delta_{M}\left(v_{i}\right)=\sum_{k: v_{i} \otimes v_{k} \in I_{v_{i}}} w_{v_{i}}\left(v_{i} \otimes v_{k}\right) v_{i} \otimes v_{k}, \\
& \tilde{\Delta}_{M}\left(v_{i}\right)=\sum_{j: v_{j} \otimes v_{i} \in J v_{i}} \tilde{w}_{v_{i}}\left(v_{j} \otimes v_{i}\right) v_{j} \otimes v_{i} .
\end{aligned}
$$

A Markov $L$-coalgebra is said to be finite when $I_{v_{i}}$ and $J_{v_{i}}$ are finite sets for all $i$. The linear maps $s, t: k^{c} \mathscr{G}_{1} \rightarrow k^{\mathscr{C}} \mathscr{G}_{0}$ given by $s\left(v_{i} \otimes v_{j}\right)=v_{i}$ and $t\left(v_{i} \otimes v_{j}\right)=v_{j}$, for all $v_{i}, v_{j} \in \mathscr{G}_{0}$, are still called source and terminus, respectively. Let $\left(\mathscr{G}, \Delta_{M}, \tilde{\Delta}_{M}\right)$ 
be a finite Markov $L$-coalgebra and $v_{i} \in \mathscr{G}_{0}$. The future of $v_{i}$ is defined as $t\left(\Delta_{M}\left(v_{i}\right)\right)$ and the past of $v_{i}$ as $s\left(\tilde{\Delta}_{M}\left(v_{i}\right)\right)$.

REMARK 3.4. The definition of a Markov $L$-coalgebra is basis-dependent. With the viewpoint of discrete Markov processes in mind, the definitions of future and past are constructed on linear superpositions of usual classical future(s) (or past(s)) and are reminiscent of quantum definitions of future and past, see [7].

THEOREM 3.5. Any weighted directed graph $G=\left(G_{0}, G_{1}, s, t\right)$, supposed to be locally finite, row-finite, without sink and source, and equipped with a family of weights $\left(w_{v}\right)_{v \in G_{0}}$, gives a finite Markov L-coalgebra.

Proof. Let $G=\left(G_{0}, G_{1}, s, t\right)$ be a directed graph, verifying Theorem 3.5. We consider the free $k$-vector space $k G_{0}$. Identify any directed arrow $v \rightarrow w$ of $G_{1}$ with $v \otimes w$. The set $G_{1}$ is then viewed as a subset of $k G_{0}^{\otimes 2}$. The family of weights $\left(w_{v}\right)_{v \in G_{0}}$ is then viewed as a family of maps $w_{v}: F_{v} \rightarrow k$, where $F_{v}:=\left\{a \in G_{1}\right.$, $s(a)=v\}$. Define the coproducts $\Delta_{M}, \tilde{\Delta}_{M}: k G_{0} \rightarrow k G_{0}^{\otimes 2}$ as follows:

$$
\begin{aligned}
& \Delta_{M}(v):=\sum_{i: a_{i} \in F_{v}} w_{v}\left(a_{i}\right) v \otimes t\left(a_{i}\right), \\
& \tilde{\Delta}_{M}(v):=\sum_{i: a_{i} \in P_{v}} w_{s\left(a_{i}\right)}\left(a_{i}\right) s\left(a_{i}\right) \otimes v,
\end{aligned}
$$

where $P_{v}:=\left\{a \in G_{1}, t(a)=v\right\}$, for all $v \in G_{0}$. For all $v \in G_{0}$, the maps $\tilde{w}_{v}$ : $P_{v} \rightarrow k$ are such that $\tilde{w}_{v}\left(a_{i}\right)=w_{s\left(a_{i}\right)}\left(a_{i}\right)$, for all $a_{i} \in P_{v}$. With these definitions the free $k$-vector space $k G_{0}$, equipped with coproducts $\Delta_{M}$ and $\tilde{\Delta}_{M}$, is a finite Markov L-coalgebra.

Motivated by this theorem, we construct a weighted directed graph from each $L$-coalgebra.

DEFINITION 3.6 (geometric support). Let $(L, \Delta, \tilde{\Delta})$ be an $L$-coalgebra generated, as a $k$-vector space, by an independent spanning set $L_{0}$. To any $v, w \in L_{0}$ such that the coefficient $\lambda$ of the element $v \otimes w$ is different from zero either in $\Delta(z)$ or in $\tilde{\Delta}(z)$, for some $z \in L_{0}$, a weighted directed arrow $v \stackrel{\lambda}{\rightarrow} w$ is associated. The weighted directed graph so obtained, denoted by $\operatorname{Gr}(L)$, is called the geometric support of $L$. Its vertex set is $L_{0}$ and its arrow set the set of those tensor products $v \otimes w, v, w \in L_{0}$, which appears in the definition of the coproducts. This construction is basis-dependent.

DEFINITION 3.7 ( $L$-cocommutator). If $\left(C, \Delta_{C}\right)$ is a coassociative coalgebra, $\operatorname{ker}\left(\Delta_{C}-\tau \Delta_{C}\right)$ represents the cocommutator subspace of $C$. Similarly, an $L^{-}$ coalgebra $(L, \Delta, \tilde{\Delta})$ is said to be $L$-cocommutative if $L^{\natural}=L$, where $L^{\natural}:=\operatorname{ker}(\Delta-$ $\tau \tilde{\Delta}$ ) is called the $L$-cocommutator subspace of $L$.

THEOREM 3.8. Let $\left(\varphi, \Delta_{M}, \tilde{\Delta}_{M}\right)$ be a finite Markov L-coalgebra generated, as a $k$-vector space, by an independent spanning set $\varphi_{0}$ with families of weights 
$\left(w_{v}\right)_{v \in \mathscr{G}_{0}}$ and $\left(w_{v}^{\prime}\right)_{v \in \mathscr{G}_{0}}$. If $v \in \mathscr{G}_{0}$ such that for each arrow $a \in \operatorname{Gr}\left(\mathscr{G}_{1}\right)_{1}$ emerging from $v$, with a given weight $w_{v}(a)$, there exists a unique arrow $b \in \operatorname{Gr}(\mathscr{G})_{1}$, such that $s(b)=t(a), t(b)=v$, and $w_{v}(a)=w_{v}^{\prime}(b)$, then $v \in G^{\natural}$.

Proof. The proof is straightforward.

REMARK 3.9. The $L$-cocommutativity describes algebraically the fact that a directed graph can be bidirected. The characterization of bidirected graphs leads naturally to constructing Leibniz bracket, see the introduction and also $[8,12]$.

THEOREM 3.10. Let $\left(\varphi, \Delta_{M}, \tilde{\Delta}_{M}\right)$ be a finite Markov L-coalgebra generated, as a $k$-vector space, by an independent spanning set $\mathscr{G}_{0}$ equipped with two families of weights $\left(w_{v}\right)_{v \in \mathscr{G}_{0}}$ and $\left(\tilde{w}_{v}\right)_{v \in \mathscr{G}_{0}}$. Suppose that for all $v \in \mathscr{G}_{0}, w_{v}$ and $\tilde{w}_{v}$ take values in $\mathbb{R}_{+}$. The family of weights $\left(w_{v}\right)_{v \in \mathscr{G}_{0}}$ describes a family of probability vectors on $\operatorname{Gr}(\mathscr{G})$ if and only if the right counit $v \mapsto \epsilon(v):=1$, for all $v \in \mathscr{G}_{0}$, exists.

Proof. The proof is straightforward.

THEOREM 3.11. Let $G=\left(G_{0}, G_{1}, s, t\right)$ be a directed graph supposed to be locally finite, rowfinite, without sink and source, and equipped with a family of probability vectors $\left(\Pi_{v}\right)_{v \in G_{0}}$. Then $G$ can be seen as the geometric support from a finite Markov L-coalgebra equipped with right and left counits.

Proof. Let $G=\left(G_{0}, G_{1}, s, t\right)$ be a directed graph verifying Theorem 3.11. We consider the free $k$-vector space $k G_{0}$. For all $v \in G_{0}$, define the right coproduct $\Delta_{M}: k G_{0} \rightarrow k G_{0}^{\otimes 2}$ such that for all $v \in G_{0}, \Delta_{M}(v)=\sum_{a \in F_{v}} \Pi_{v}(a) v \otimes$ $t(a)$, the left coproduct $\tilde{\Delta}_{M}: k G_{0} \rightarrow k G_{0}^{\otimes 2}$ verifying for all $v \in G_{0}, \tilde{\Delta}_{M}(v)=$ $\left(1 / \operatorname{card}\left(P_{v}\right)\right) \sum_{a \in P_{v}} s(a) \otimes v$, and the right (resp., the left) counit $\epsilon$ (resp., $\tilde{\epsilon}$ ) such that $\tilde{\epsilon}(v)=1=\epsilon(v)$ for all $v \in G_{0}$. This finite Markov $L$-coalgebra has two counits and its geometric support is $G$.

Proposition 3.12. Let $\left(\varphi, \Delta_{M}, \tilde{\Delta}_{M}\right)$ be a finite Markov L-coalgebra generated, as a $k$-vector space, by an independent spanning set $\mathscr{G}_{0}$. The sequence $\left(\Delta_{M}\right)_{1} \equiv \Delta_{M},\left(\Delta_{M}\right)_{2}=\mathrm{id} \otimes \Delta_{M},\left(\Delta_{M}\right)_{3}=\mathrm{id} \otimes \mathrm{id} \otimes \Delta_{M}, \ldots$ generates all possible weighted paths in $\mathrm{Gr}(\mathscr{G})$, starting at any vertex. Similarly, the sequence $\left(\tilde{\Delta}_{M}\right)_{n \geq 0}$ generates all the possible weighted paths in $\operatorname{Gr}(\mathscr{G})$, arriving at a given vertex.

Proof. The proof is straightforward.

The algebraic framework of finite Markov $L$-coalgebras extends the classical setting of weighted directed graphs. Usually, a directed graph $G=\left(G_{0}, G_{1}, s, t\right)$ is also coded through an adjacency matrix, that is, a square matrix $A_{G}$, with $A_{G}\left[v_{i}, v_{j}\right]=1$ if the directed arrow $v_{i} \rightarrow v_{j} \in G_{1}$. With the viewpoint of random walk on directed graphs, the adjacency matrix codes the (Markovian) neighbourhood of a given vertex, that is, vertices present in the sum $t \Delta_{M}$ for the future and in the sum $s \tilde{\Delta}_{M}$ for the past, when we view the graph through its 


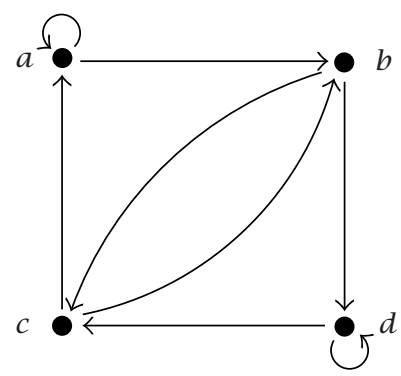

FIGURE 3.1. Directed graph associated with $\mathrm{Sl}_{q}(2)$.

Markov $L$-coalgebra. The coproducts $\Delta_{M}$ and $\tilde{\Delta}_{M}$ can be called the propagators on the geometric support of the Markov $L$-coalgebra. The locality is respected. If $v_{1}, \ldots, v_{n}$ denote the vertices of the graph $G$, then observe that $t\left(\Delta_{M}(v)\right)=A_{G} \cdot X$, where the adjacency matrix $A_{G}$ is written in the basis $\left(v_{1}, \ldots, v_{n}\right)$, and $X$ is the vector ${ }^{t} v:={ }^{t}\left(\lambda_{1}, \ldots, \lambda_{n}\right)$ in the same basis.

Motivated by this framework, for any $L$-coalgebra $(L, \Delta, \tilde{\Delta})$ generated, as a $k$-vector space, by an independent spanning set $L_{0}$, a unique directed graph $\operatorname{Gr}(L)$, called the geometric support of $L$, has been constructed. Regarding the coproducts of $L$ as a propagator of a walk generated by the sequences $\left(\Delta_{n}\right)_{n>0}$ and $\left(\tilde{\Delta}_{n}\right)_{n>0}$, we define the future of $v \in L_{0}$ by $t(\Delta(v))$ and the past by $s(\tilde{\Delta}(v))$. We see what occurs in the case of a coassociative coalgebra.

First of all, we enlarge our graphical construction to bialgebra. Let ( $A:=$ $\left.k\left\{A_{0}\right\} \backslash \mathscr{R}, \Delta, m, \eta\right)$ be a bialgebra such that $\left(A:=k\left\{A_{0}\right\} \backslash \mathscr{R}, m, \eta\right)$ is an associative algebra generated by a denumerable set $A_{0}$ verifying a set of relations $\mathscr{R}$. Consider the subvector space of $A$ spanned by the set $A_{0}$ and denoted by $k A_{0}$. If $A_{0}$ is an independent spanning set and $\Delta: k A_{0} \rightarrow k A_{0}^{\otimes 2}$, then we construct its geometric support as before. In other terms, the vertex set is composed by the generators of the algebra $A$ and the arrows still represent the tensor products appearing in the definition of $\Delta$. Fix an invertible element $q \in k$. The Hopf algebra $\mathrm{Sl}_{q}(2)$ is generated by $a, b, c$, and $d$ such that $b a=q a b, c a=q a c$, $d c=q c d, d b=q b d, b c=c b, a d-d a=\left(q^{-1}-q\right) b c$, and $a d-q^{-1} b c=1$. The antipode map is described by the linear map $S: \operatorname{Sl}_{q}(2) \rightarrow \operatorname{Sl}_{q}(2)$ such that $S(a)=d, S(d)=a, S(b)=-q b$, and $S(c)=-q^{-1} c$. The well-known coalgebra structure is described by $\Delta_{\mathrm{Sl}}(a)=a \otimes a+b \otimes c, \Delta_{\mathrm{Sl}}(b)=a \otimes b+b \otimes d$, $\Delta_{\mathrm{Sl}}(c)=d \otimes c+c \otimes a$, and $\Delta_{\mathrm{Sl}}(d)=d \otimes d+c \otimes b$. The directed graph associated with $\mathrm{Sl}_{q}(2)$ is illustrated in Figure 3.1.

The geometric support of $\mathrm{Sl}_{q}(2)$, whose future and past of a given vertex are coded by its coproduct $\Delta_{\mathrm{Sl}}$, behaves in a nonlocal way. For the sake of an example, notice that on the directed graph $\mathrm{Gr}\left(\mathrm{Sl}_{q}(2)\right)$, the future of $a$ is not $a$ and $b$ as expected in usual graph theory. On the contrary, the future of $a$ is $a$ and $c$ and its past is $a$ and $b$. 
Observe also that the antipode map, as an anticoalgebra map, has also an interesting interpretation since it realizes a time reversal. (The future becomes the past and conversely.)

PROPOSITION 3.13. If $\left(C, \Delta_{C}\right)$ is a cocommutative coassociative coalgebra generated, as a $k$-vector space, by an independent spanning set $C_{0}$, then its geometric support $\operatorname{Gr}(C)$ can be viewed as a nondirected graph.

Proof. Let $\left(C, \Delta_{C}\right)$ be a cocommutative coassociative coalgebra generated, as a $k$-vector space, by an independent spanning set $C_{0}$. Let $a, b, x \in C_{0}$ and suppose that the term $a \otimes b$ appears in the description of $\Delta x$. The same must be true for $b \otimes a$ since $\Delta_{C}=\tau \Delta_{C}$. On the geometric support $\operatorname{Gr}(C)$, an arrow emerges from $a$ to $b$ and from $b$ to $a$. We have just proved that the graph is bidirected. By identifying the arrow emerging from $a$ to $b$ with that from $b$ to $a$, we obtain a nondirected graph.

To embed any directed graph into an algebraic framework, we use the formalism of $L$-coalgebra. This point of view has the advantage to manipulate weighted directed graphs and deals with future and past in an algebraic way and to generalize these notions to any $L$-coalgebra. We mention that any directed graph can also be embedded into a coassociative coalgebra by considering its path space instead of its vertex space [1]. This result can also be recovered by the following theorem.

THEOREM 3.14. Let $G$ be a nonempty set consisting of a distinguished subset $G^{(0)} \subset G$, two maps $t, s: G \rightarrow G^{(0)}$, and a law of composition

$$
\circ: G^{(2)}=\left\{\left(\gamma_{1}, \gamma_{2}\right) \in G \times G ; s\left(\gamma_{1}\right)=t\left(\gamma_{2}\right)\right\} \longrightarrow G
$$

such that

(1) $s\left(\gamma_{1} \circ \gamma_{2}\right)=s\left(\gamma_{2}\right), t\left(\gamma_{1} \circ \gamma_{2}\right)=t\left(\gamma_{1}\right)$, for all $\left(\gamma_{1}, \gamma_{2}\right) \in G^{(2)}$,

(2) for all $x \in G^{(0)}, s(x)=t(x)=x$; for all $\gamma \in G, \gamma \circ s(\gamma)=\gamma, t(\gamma) \circ \gamma=\gamma$,

(3) $\left(\gamma_{1} \circ \gamma_{2}\right) \circ \gamma_{3}=\gamma_{1} \circ\left(\gamma_{2} \circ \gamma_{3}\right)$,

(4) the family $\left(G_{\gamma}:=\left\{\left(\gamma_{1}, \gamma_{2}\right) \in G^{(2)} ; \gamma=\gamma_{1} \circ \gamma_{2}\right\}\right)_{\gamma \in G}$ is a family of finite sets.

Let $C$ be a k-vector space equipped with a right action $\alpha: C \times G \rightarrow C$. If $\tilde{C}:=$ $\left\{c_{\gamma}=\alpha(c, \gamma), \gamma \in G\right\}$ is the $k$-vector space of orbits associated with $\alpha$, then $\tilde{C}$ has a coassociative coalgebra structure.

Proof. Fix $c_{\gamma} \in \tilde{C}$ and define $\Delta c_{\gamma}:=\sum_{\gamma_{1} \circ \gamma_{2}=\gamma} c_{\gamma_{1}} \otimes c_{\gamma_{2}}$. From condition (2), one gets that there exists at least an element $\left(\gamma_{1}, \gamma_{2}, \gamma_{3}\right) \in G^{\times 3}$ such that $\gamma=$ $\gamma_{1} \circ \gamma_{2} \circ \gamma_{3}$. By the definition of the coproduct and the associativity of the product $\circ$, we get

$$
\begin{aligned}
\sum_{\gamma_{1} \circ \gamma_{2}=\gamma} c_{\gamma_{1}} \otimes \Delta\left(c_{\gamma_{2}}\right) & =\sum_{\gamma_{1} \circ \gamma_{2}=\gamma} \sum_{\gamma_{1}^{\prime} \circ \gamma_{2}^{\prime}=\gamma_{2}} c_{\gamma_{1}} \otimes\left(c_{\gamma_{1}^{\prime}} \otimes c_{\gamma_{2}^{\prime}}\right) \\
& =\sum_{\gamma_{1} \circ\left(\gamma_{1}^{\prime} \circ \gamma_{2}^{\prime}\right)=\gamma} c_{\gamma_{1}} \otimes\left(c_{\gamma_{1}^{\prime}} \otimes c_{\gamma_{2}^{\prime}}\right),
\end{aligned}
$$




$$
\begin{aligned}
\sum_{\gamma_{1} \circ \gamma_{2}=\gamma} \Delta\left(c_{\gamma_{1}}\right) \otimes c_{\gamma_{2}} & =\sum_{\gamma_{1} \circ \gamma_{2}=\gamma} \sum_{\gamma_{1}^{\prime \prime} \circ \gamma_{2}^{\prime \prime}=\gamma_{1}}\left(c_{\gamma_{1}^{\prime \prime}} \otimes c_{\gamma_{2}^{\prime \prime}}\right) \otimes c_{\gamma_{2}} \\
& =\sum_{\left(\gamma_{1}^{\prime \prime} \circ \gamma_{2}^{\prime \prime}\right) \circ \gamma_{2}=\gamma}\left(c_{\gamma_{1}^{\prime \prime}} \otimes c_{\gamma_{2}^{\prime \prime}}\right) \otimes c_{\gamma_{2}},
\end{aligned}
$$

proving that $($ id $\otimes \Delta) \Delta=(\Delta \otimes$ id $) \Delta$ since the sums involved are over all possible decompositions of $\gamma$ in three parts. As $\gamma=\gamma \circ s(\gamma)=t(\gamma) \circ \gamma$, we define $\epsilon\left(c_{\gamma}\right)=$ 0 if $\gamma \in G \backslash G^{(0)}$ and $\epsilon\left(c_{\gamma}\right)=1$ otherwise. We have

$$
\Delta\left(c_{\gamma}\right)=\sum_{\gamma_{1} \circ \gamma_{2}=\gamma ; \gamma \in G \backslash G^{(0)}} c_{\gamma_{1}} \otimes c_{\gamma_{2}}+c_{t(\gamma)} \otimes c_{\gamma}+c_{\gamma} \otimes c_{s(\gamma)},
$$

thus $(\mathrm{id} \otimes \epsilon) \Delta=(\epsilon \otimes \mathrm{id}) \Delta=\mathrm{id}$.

REMARK 3.15. Observe that a directed graph, as a geometric object, can be a geometric support for several $L$-coalgebras. For instance the geometric support of the coassociative coalgebra $(\mathscr{F}, \Delta)$, spanned as a $k$-vector space by a basis $a, b, c$, and $d$ and whose coproduct $\Delta$ is given by $\Delta(a)=a \otimes a+b \otimes c$, $\Delta(b)=a \otimes b+b \otimes d, \Delta(c)=d \otimes c+c \otimes a$, and $\Delta(d)=d \otimes d+c \otimes b$, is the same as, or isomorphic to, the geometric support of the finite Markov $L$-coalgebra spanned, as a $k$-vector space, by a basis $a, b, c$, and $d$ and described by the right coproduct $\Delta_{M}(a)=a \otimes(a+b), \Delta_{M}(b)=b \otimes(c+d), \Delta_{M}(c)=c \otimes(a+b)$, $\Delta_{M}(d)=d \otimes(c+d)$ and the left coproduct $\tilde{\Delta}_{M}(a)=(a+c) \otimes a, \tilde{\Delta}_{M}(b)=(a+$ $c) \otimes b, \tilde{\Delta}_{M}(c)=(b+d) \otimes c, \tilde{\Delta}_{M}(d)=(b+d) \otimes d$.

To turn a coassociative coalgebra into a nondegenerate $L$-coalgebra, we will use a generalization of an idea applied by Hudson [4].

Proposition 3.16. Let $\left(C, \Delta_{C}\right)$ be a coassociative coalgebra with a grouplike element $e$. Define the coproducts $\tilde{\delta}_{f}, \delta_{f}: C \rightarrow C^{\otimes 2}$ such that for all $c \in C$, $\tilde{\delta}_{f}(c):=e \otimes c$ and $\delta_{f}(c):=c \otimes e$. Then $\left(C, \tilde{\delta}_{f}, \delta_{f}\right)$ is a finite Markov L-coalgebra which is in addition a coassociative codialgebra.

PROOF. The proof is straightforward.

Proposition 3.17. Any coassociative coalgebra $\left(C, \Delta_{C}\right)$, with a group-like element, gives rise to a nondegenerate $L$-coalgebra structure on $C$.

Proof. Let $\left(C, \Delta_{C}\right)$ be a coassociative coalgebra. Suppose $e$ is a group-like element, that is, $\Delta_{C}(e)=e \otimes e$. Define, as in Proposition 3.16, the coassociative coproducts $\delta_{f}(c):=c \otimes e$ and $\tilde{\delta}_{f}(c):=e \otimes c$, for all $c \in C$. Define also two linear maps $\vec{d}, \overleftarrow{d}: C \rightarrow C \otimes C$ by $\vec{d}(c)=\Delta_{C}(c)-\delta_{f}(c)$ and $\overleftarrow{d}(c)=\Delta_{C}(c)-\tilde{\delta}_{f}(c)$, for all $c \in C$. These linear maps $\overleftarrow{d}$ and $\vec{d}$ turn the coassociative coalgebra $C$ into a nondegenerate $L$-coalgebra. Indeed, if $c \in C$ such that $\Delta_{C}(c)=\sum c_{(1)} \otimes c_{(2)}$, 
then

$$
\begin{aligned}
c & \stackrel{\bar{d}}{\longrightarrow} \sum c_{(1)} \otimes c_{(2)}-e \otimes c \\
& \stackrel{\mathrm{id} \otimes \vec{d}}{\longrightarrow} \sum c_{(1)} \otimes \Delta_{C}\left(c_{(2)}\right)-\Delta_{C}(c) \otimes e-e \otimes \Delta_{C}(c)+e \otimes c \otimes e, \\
c & \stackrel{\vec{d}}{\longrightarrow} \sum c_{(1)} \otimes c_{(2)}-c \otimes e \\
& \stackrel{\bar{d} \otimes \mathrm{id}}{\longrightarrow} \sum \Delta_{C}\left(c_{(1)}\right) \otimes c_{(2)}-\Delta_{C}(c) \otimes e-e \otimes \Delta_{C}(c)+e \otimes c \otimes e .
\end{aligned}
$$

Moreover, $\overleftarrow{d}$ is obviously not equal to $\vec{d}$ on the whole coalgebra. Therefore, $(C, \vec{d}, \overleftarrow{d})$ is a nondegenerate $L$-coalgebra.

REMARK 3.18. Let $\left(C, \Delta_{C}, \epsilon_{C}\right)$ be a coassociative coalgebra with counit $\epsilon_{C}$. Observe that $c \in C \cap \operatorname{ker} \epsilon_{C}$ and $(\overleftarrow{d} \otimes \mathrm{id}) \vec{d}(c)=0$ if and only if $c$ is a primitive element of $C$.

COROLLARY 3.19. Any bialgebra can be viewed as a nontrivial L-coalgebra.

PROoF. The proof is straightforward since the identity element is grouplike.

We can construct another interesting class of $L$-coalgebra.

DEFINITION 3.20 ( $C$-bimodule). Let $\left(C, \Delta_{C}\right)$ be a bialgebra. From Proposition $3.17,(C, \overleftarrow{d}, \vec{d})$ is an $L$-coalgebra. Define on $C^{\otimes 2}$ two structures of $C$-bimodule given by the following products: for $x, y \in C, c \in C^{\otimes 2}, x \tilde{x}^{\tilde{c}}=\tilde{\delta}_{f}(x) c, c \tilde{*} y=$ $c \tilde{\delta}_{f}(y), x \cdot c=\delta_{f}(x) c$, and $c \cdot y=c \delta_{f}(y)$.

Let $A$ be an algebra with unit 1 and $M$ an $A$-bimodule. A Leibniz-Ito derivative is a linear map $\rho: A \rightarrow M$ such that for all $x, y \in A, \rho(x y)=x \rho(y)+\rho(x) y+$ $\rho(x) \rho(y)$ and $\rho(1)=0$.

THEOREM 3.21. Let $\left(C, \Delta_{C}\right)$ be a bialgebra. As $\Delta_{C}$ is a unital homomorphism, the coproducts $\overleftarrow{d}$ and $\vec{d}$ turn out to be Leibniz-Ito derivatives.

Proof. Let $\left(C, \Delta_{C}\right)$ be a bialgebra and $x, y \in C$. The relation $\overleftarrow{d}(1)=0=$ $\vec{d}(1)$ holds. Moreover, $\vec{d}(x) \vec{d}(y)=\Delta_{C}(x y)+x y \otimes 1-\Delta_{C}(x)(y \otimes 1)-(x \otimes$ 1) $\Delta(y)$ implies $\vec{d}(x y)=\vec{d}(x) \vec{d}(y)+\vec{d}(x) \cdot y+x \cdot \vec{d}(y)$. The same equation holds for the other coproduct.

If $\left(C, \Delta_{C}\right)$ is a bialgebra, we call $(C, \overleftarrow{d}, \vec{d})$ a Leibniz-Ito L-coalgebra. This kind of $L$-coalgebra plays an important role in quantum stochastic processes, see $[3,4]$.

We present here two ways to construct $L$-coalgebras from known ones.

3.1.1. Bicomodule over $C$ for a coassociative coalgebra $C$. Let $\left(C, \Delta_{C}\right)$ be a coassociative coalgebra. Let $B$ be a $C$-bicomodule, that is, there exist linear 
maps $\delta$ and $\tilde{\delta}$ such that the following diagram commutes:

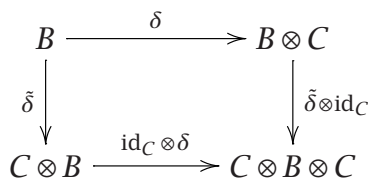

and such that $\left(\operatorname{id}_{B} \otimes \Delta_{C}\right) \delta=\left(\delta \otimes \operatorname{id}_{C}\right) \delta$ and $\left(\Delta_{C} \otimes \operatorname{id}_{B}\right) \tilde{\delta}=\left(\operatorname{id}_{C} \otimes \tilde{\delta}\right) \tilde{\delta}$.

Proposition 3.22. Let $\left(C, \Delta_{C}\right)$ be a coassociative coalgebra and $B$ a bicomodule over $C$. Let $A:=C \otimes B \otimes C$. Keeping the previous notation, the linear maps $\delta$ and $\tilde{\delta}$ induce coproducts $\Delta$ and $\tilde{\Delta}$ on $A$ given by

$$
\begin{aligned}
& \Delta(u \otimes b \otimes v):=(u \otimes \delta(b)) \otimes(v \otimes e \otimes f), \\
& \tilde{\Delta}(u \otimes b \otimes v):=(g \otimes h \otimes u) \otimes(\tilde{\delta}(b) \otimes v),
\end{aligned}
$$

for $u \otimes b \otimes v \in A$ and fixed elements $g, f \in C$ and $h, e \in B$. That means that there exists a natural structure of L-coalgebra on A for any couple of pairs $(g, f) \in C^{\times 2}$ and $(h, e) \in B^{\times 2}$.

Proof. Let $\left(C, \Delta_{C}\right)$ be a coassociative coalgebra and $B$ a bicomodule over $C$. Fix $g, f \in C$ and $h, e \in B$. Set $A:=C \otimes B \otimes C$. The coproducts $\delta$, $\delta$ induce coproducts $\Delta, \tilde{\Delta}$ defined above such that the following diagram commutes:

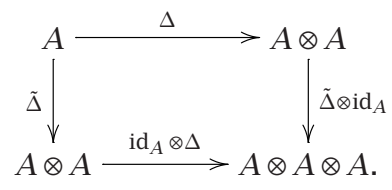

Indeed,

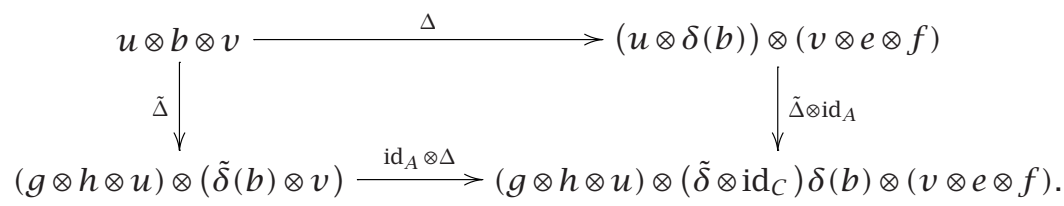

3.1.2. Tensor product. Let $\left(L, \Delta_{L}, \tilde{\Delta}_{L}\right)$ and $\left(M, \Delta_{M}, \tilde{\Delta}_{M}\right)$ be two $L$-coalgebras. Define the right coproduct $\Delta_{L \otimes M}$ to be the composite

$$
L \otimes M \stackrel{\Delta_{L} \otimes \Delta_{M}}{\longrightarrow}(L \otimes L) \otimes(M \otimes M) \stackrel{\operatorname{id}_{L} \otimes T \otimes \operatorname{id}_{M}}{\longrightarrow}(L \otimes M) \otimes(L \otimes M)
$$

and the left coproduct $\tilde{\Delta}_{L \otimes M}$ by

$$
L \otimes M \stackrel{\tilde{\Delta}_{L} \otimes \tilde{\Delta}_{M}}{\longrightarrow}(L \otimes L) \otimes(M \otimes M) \stackrel{\operatorname{id}_{L} \otimes T \otimes \mathrm{id}_{M}}{\longrightarrow}(L \otimes M) \otimes(L \otimes M) .
$$


With this setting, $\left(L \otimes M, \Delta_{L \otimes M}, \tilde{\Delta}_{L \otimes M}\right)$ becomes an $L$-coalgebra over $k$. If both $L$ coalgebras have counits, we define the right counit $\epsilon_{L \otimes M}$ as $L \otimes M \stackrel{\epsilon_{L} \otimes \epsilon_{M}}{\longrightarrow} k \otimes k \simeq$ $k$ and the left counit $\tilde{\epsilon}_{L \otimes M}$ as $L \otimes M \stackrel{\tilde{\epsilon}_{L} \otimes \tilde{\epsilon}_{M}}{\longrightarrow} k \otimes k \simeq k$.

3.2. $L$-Bialgebras and $L$-Hopf algebras. The construction of vector spaces equipped with two coproducts entails the generalization of definitions such as bialgebras and Hopf algebras.

DEFINITION 3.23 ( $L$-bialgebra). An $L$-bialgebra with counits $\epsilon$ and $\tilde{\epsilon}$ is an $L$ coalgebra with counits $(L, \Delta, \tilde{\Delta}, \epsilon, \tilde{\epsilon})$ equipped with an extra structure of unital algebra over $k$ such that the coproducts and counits are algebra homomorphisms.

DEFINITION 3.24 (L-Hopf algebra). An $L$-Hopf algebra is an $L$-bialgebra with counits $(H, \Delta, \tilde{\Delta}, \epsilon, \tilde{\epsilon})$ equipped with two linear maps $S, \tilde{S}: H \rightarrow H$, called right and left antipodes, which verify the equalities $m($ id $\otimes S) \Delta=\eta \epsilon$ and $m(\tilde{S} \otimes$ id) $\tilde{\Delta}=\eta \tilde{\epsilon}$.

REMARK 3.25. In the sequel, all our $L$-Hopf algebras will verify the previous equalities. However, it is worth noticing [8] that we can also construct another type of $L$-Hopf algebras verifying the equalities $m(S \otimes$ id $) \Delta=\eta \epsilon$ and $m(\mathrm{id} \otimes \tilde{S}) \tilde{\Delta}=\eta \tilde{\epsilon}$.

We give two examples of $L$-bialgebras.

EXAMPLE 3.26 (the Cuntz-Krieger algebra). In [2, 6], a $C^{*}$-algebra, called a Cuntz-Krieger algebra, is associated with each directed graph $G=\left(G_{0}, G_{1}, s, t\right)$. If $G$ is a row-finite (i.e., for all $v \in G_{0}, s^{-1}(\{v\})$ is finite) directed graph, a Cuntz-Krieger $G$-family consists of a set $\left\{P_{v}: v \in G_{0}\right\}$ of mutually orthogonal projections and a set $\left\{S_{e}: e \in G_{1}\right\}$ of partial isometries satisfying

$$
S_{e}^{*} S_{e}=P_{t(e)}, \quad P_{v}=\sum_{e: s(e)=v} S_{e} S_{e}^{*}, \quad \forall(e, v) \in G_{1} \times G_{0},
$$

where, for all $e \in G_{1}, S_{e}^{*}$ denotes the adjoint of $S_{e}$.

Proposition 3.27. A Cuntz-Krieger algebra CK associated with a graph without sinks and loops and whose vertex set is finite is a finite Markov Lbialgebra.

Proof. As usual, by defining

$$
\begin{aligned}
& \Delta_{M}\left(P_{v}\right)=\sum_{v_{1} \in t\left(s^{-1}(\{v\})\right)} P_{v} \otimes P_{v_{1}}, \\
& \tilde{\Delta}_{M}\left(P_{v}\right)=\sum_{v_{0} \in S\left(t^{-1}(\{v\})\right)} P_{v_{0}} \otimes P_{v},
\end{aligned}
$$



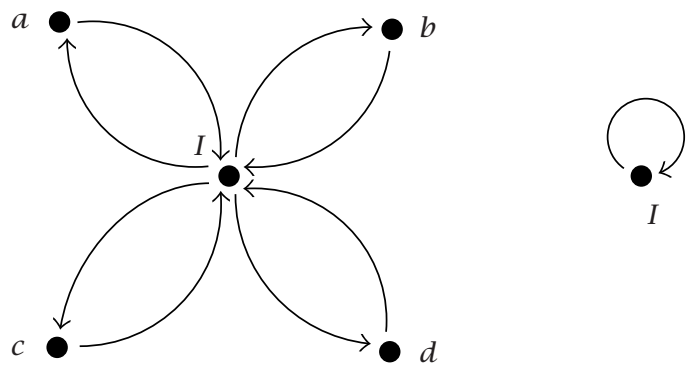

FIGURE 3.2. Example of geometric support associated with an algebra $k\{a, b, c, d\} \oplus k I$.

we turn CK into a Markov L-coalgebra. Thanks to the mutual orthogonality property of the projectors, we get, for instance,

$$
\begin{aligned}
\Delta_{M}\left(P_{v}\right) \Delta_{M}\left(P_{v^{\prime}}\right) & =\sum_{v_{1} \in t\left(s^{-1}(v)\right)} \sum_{v_{1}^{\prime} \in t\left(s^{-1}\left(v^{\prime}\right)\right)} P_{v} P_{v^{\prime}} \otimes P_{v_{1}} P_{v_{1}^{\prime}} \\
& =\delta\left(v, v^{\prime}\right) \Delta_{M}\left(P_{v}\right)=\Delta_{M}\left(P_{v} P_{v^{\prime}}\right)
\end{aligned}
$$

where $\delta$ is the Kronecker symbol. Since the vertex set is finite, CK has an identity element $\sum_{v \in G_{0}} P_{v}:=I$. In general, we do not have $\Delta_{M}(I)=I \otimes I=\tilde{\Delta}_{M}(I)$.

EXAMPLE 3.28 (unital algebra). Let $A$ be a unital algebra with unit $I$. From the equality $(I \cdot a) \cdot I=I \cdot(a \cdot I)$, A carries a nontrivial finite Markov $L$-bialgebra, with coproducts $\delta_{f}(a)=a \otimes I$ and $\tilde{\delta}_{f}(a)=I \otimes a$, for all $a \in A$. If $A:=k\left\{A_{0}\right\} \backslash \mathscr{R}$ is an associative algebra generated by a denumerable set $A_{0}$ verifying a set of relations $\mathscr{R}$, then its geometric support can be constructed. We call it the flower graph (see Figure 3.2).

Observe that for all $a \in A$ different from $I, a \mapsto \delta_{f}(a)+\tilde{\delta}_{f}(a)$ and $I \mapsto I \otimes I$ is a coassociative cocommutative coproduct.

4. Finite Markov $L$-coalgebra and periodic orbits. Propositions 3.16 and 3.17 assert that a coassociative coalgebra $\left(C, \Delta_{C}\right)$ with a group-like element $e$ yields two coproducts $\overleftarrow{d}$ and $\vec{d}$, constructed from the coproduct $\Delta_{C}$, which turn out to be Leibniz-Ito derivatives if $\Delta_{C}$ is a unital homomorphism and if $e=$ $I$. In this framework, if we replace a coassociative coalgebra by a finite Markov $L$-coalgebra, how can we produce two new coproducts such that if the old ones are unital homomorphisms, the new ones become Leibniz-Ito derivatives?

The answer to this question can be found, in the proof of Proposition 3.17, by analysing the term $e \otimes x \otimes e$. Graphically, this term describes the path $e \rightarrow x \rightarrow e$, that is, we have made one complete turn around the orbit $(e, x, e)$ of the flower graph. In a general finite Markov $L$-coalgebra $\mathscr{G}$ generated by an independent spanning set $\mathscr{G}_{0}$, there does not exist such a possibility. Therefore we have 
to create it. To do so, we need to consider the arrow set $\mathscr{G}_{1}$ of the geometric support of a finite Markov $L$-coalgebra $\mathscr{G}$ and fix an arrow, say $a \rightarrow b$, which is associated with $a \otimes b$ in $G^{\otimes 2}$. By this way, we can construct two virtual periodic orbits of period 2, either $a \rightarrow b \rightarrow a$ or $b \rightarrow a \rightarrow b$. Before going on, we introduce a notion inspired from the line extension of graph: the $L$-coalgebras of degree $n$

Definition 4.1. Let $n \in \mathbb{N} \backslash\{0\}$. The $k$-vector space $\left(Z, \Delta_{n}, \tilde{\Delta}_{n}\right)$ is an $L$ coalgebra of degree $n$ over $k$ if the diagram

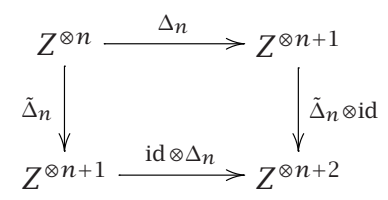

commutes, that is, $\left(\tilde{\Delta}_{n} \otimes\right.$ id $) \Delta_{n}=\left(\right.$ id $\left.\otimes \Delta_{n}\right) \tilde{\Delta}_{n}$. Such a space may have a right counit $\epsilon_{n}: Z^{\otimes n} \rightarrow Z^{\otimes n-1}$ such that $\left(\mathrm{id} \otimes \epsilon_{n}\right) \Delta_{n}=$ id and a left counit $\tilde{\epsilon}_{n}: Z^{\otimes n} \rightarrow$ $Z^{\otimes n-1}$ such that $\left(\tilde{\epsilon}_{n} \otimes \mathrm{id}\right) \tilde{\Delta}_{n}=\mathrm{id}$. By convention, $Z^{\otimes 0}:=k$.

Proposition 4.2. A finite Markov L-coalgebra $\left(\varphi, \Delta_{M}, \tilde{\Delta}_{M}\right)$ is a finite Markov $L$-coalgebra of degree $n$ for any $n>0$.

Proof. Let $\Delta_{M}, \tilde{\Delta}_{M}, \epsilon$, and $\tilde{\epsilon}$ be the coproducts and the possible counits of a finite Markov $L$-coalgebra $\mathscr{G}$ and define the following operators:

$$
\begin{gathered}
\left(\Delta_{M}\right)_{n}=\underbrace{\mathrm{id} \otimes \cdots \otimes \mathrm{id}}_{n-1} \otimes \Delta_{M}, \quad\left(\tilde{\Delta}_{M}\right)_{n}=\tilde{\Delta}_{M} \otimes \underbrace{\mathrm{id} \otimes \cdots \otimes \mathrm{id}}_{n-1}, \\
\epsilon_{n}=\underbrace{\mathrm{id} \otimes \cdots \otimes \mathrm{id}}_{n-1} \otimes \epsilon, \quad \tilde{\epsilon}_{n}=\tilde{\epsilon} \otimes \underbrace{\mathrm{id} \otimes \cdots \otimes \mathrm{id}}_{n-1} .
\end{gathered}
$$

Equipped with these maps, $\mathscr{G}$ is a finite Markov $L$-coalgebra of degree $n$.

From now on, we consider the special case $n=2$. The Markov $L$-coalgebra $\mathscr{G}$ can be embedded into a Markov $L$-coalgebra of degree 2 . We see now the link with the line extension in graph theory. Let $\left(\mathscr{G}, \Delta_{M}, \tilde{\Delta}_{M}\right)$ be a Markov $L^{-}$ coalgebra generated by an independent spanning set $\varphi_{0}$, whose geometric support is denoted by $\operatorname{Gr}(\mathscr{G})$. Fix $u \rightarrow v \in \operatorname{Gr}(\mathscr{G})_{1}$. Then $\left(\Delta_{M}\right)_{2}(u \otimes v):=u \otimes \Delta_{M}(v)$. Therefore, the line extension of $\operatorname{Gr}(\mathscr{G})$, denoted by $E(\operatorname{Gr}(\mathscr{G}))$, is a Markov $L^{-}$ coalgebra with coproduct $\Delta_{E}(u \otimes v):=(u \otimes v) \otimes \Delta_{M}(v)$. Recall that $s$ is the source map. Therefore, (id $\otimes S \otimes$ id) $\Delta_{E}:=\left(\Delta_{M}\right)_{2}$ since (id $\otimes S \otimes$ id) $\Delta_{E}(u \otimes v):=$ $u \otimes s(v \otimes v) \otimes t\left(\Delta_{M}(v)\right):=u \otimes \Delta_{M}(v):=\left(\Delta_{M}\right)_{2}(u \otimes v)$.

DEFINITION 4.3. Let $\varphi$ be a $k$-vector space generated by an independent spanning set $\varphi_{0}$. Define the coproducts $\delta_{R}, \delta_{L}: \varphi^{\otimes 2} \rightarrow \varphi^{\otimes 3}$, such that $\delta_{R}(a \otimes$ $b)=a \otimes b \otimes a$ and $\delta_{L}(a \otimes b)=b \otimes a \otimes b$, for all $a, b \in \varphi_{0}$.

Proposition 4.4. Let $G$ be a $k$-vector space generated by an independent spanning set $\varphi_{0}$. The coproducts $\delta_{L}$ and $\delta_{R}$ verify the coassociativity breaking 
equation $\left(\delta_{L} \otimes \mathrm{id}\right) \delta_{R}=\left(\mathrm{id} \otimes \delta_{R}\right) \delta_{L}$. Moreover, $\delta_{L}$ and $\delta_{R}$ are both homomorphisms if $G$ has also an extra structure of algebra over $k$.

Proof. Let $\varphi$ be a $k$-vector space generated by an independent spanning set $\mathscr{G}_{0}$. Fix $a, b \in \mathscr{G}_{0}$. We get $a \otimes b \stackrel{\delta_{L}}{\longrightarrow} b \otimes a \otimes b \stackrel{\text { id } \otimes \delta_{R}}{\longrightarrow} b \otimes(a \otimes b \otimes a)$ and $a \otimes b \stackrel{\delta_{R}}{\longrightarrow}$ $a \otimes b \otimes a \stackrel{\delta_{L} \otimes \text { id }}{\longrightarrow}(b \otimes a \otimes b) \otimes a$. If $\mathscr{G}_{\text {is }}$ also an algebra generated by $\mathscr{G}_{0}$, fix $(a, b, c, d) \in \varphi_{0}$, then $\delta_{L}(a \otimes b) \delta_{L}(c \otimes d)=(b \otimes a \otimes b)(d \otimes c \otimes d)=(b d \otimes a c \otimes$ $b d)=\delta_{L}(a c \otimes b d)=\delta_{L}((a \otimes b)(c \otimes d))$. The same computation is used for proving that $\delta_{R}$ is a homomorphism.

THEOREM 4.5. Let $G$ be a finite Markov L-coalgebra generated, as a $k$-vector space, by an independent spanning set $\varphi_{0}$, equipped with coproducts $\Delta_{M}$ and $\tilde{\Delta}_{M}$. Set $\left(\Delta_{M}\right)_{2}:=\mathrm{id} \otimes \Delta_{M}$ and $\left(\tilde{\Delta}_{M}\right)_{2}:=\tilde{\Delta}_{M} \otimes \mathrm{id}$. Define the two coproducts $\overleftarrow{d}_{\varphi_{G}}$ $\vec{d}_{\varphi}$ as $\overleftarrow{d}_{\varphi}=\left(\tilde{\Delta}_{M}\right)_{2}-\delta_{L}$ and $\vec{d}{ }_{\varphi}=\left(\Delta_{M}\right)_{2}-\delta_{R}$. These two coproducts verify the coassociativity breaking equation $\left(\overleftarrow{d}_{\varphi} \otimes \mathrm{id}\right) \vec{d}_{\varphi}=\left(\mathrm{id} \otimes \vec{d}_{\varphi}\right) \overleftarrow{d}_{\varphi}$. Moreover, $\vec{d}_{\varphi}=$ $0=\overleftarrow{d}_{\varphi}$ on (trivial weighted) isolated periodic orbits of period 2.

Proof. The proof is straightforward by noticing that $\left(\delta_{L} \otimes \mathrm{id}\right)\left(\Delta_{M}\right)_{2}=(\mathrm{id} \otimes$ $\left.\left(\Delta_{M}\right)_{2}\right) \delta_{L}$ and that $\left(\left(\tilde{\Delta}_{M}\right)_{2} \otimes \mathrm{id}\right) \delta_{R}=\left(\mathrm{id} \otimes \delta_{R}\right)\left(\tilde{\Delta}_{M}\right)_{2}$. Let $x, y \in \mathscr{G}_{0}$ representing a (trivial weighted) isolated periodic orbit of period 2 on $\operatorname{Gr}(\varphi)$. Such an orbit verifies that $\left(\Delta_{M}\right)_{2}(x \otimes y)=x \otimes y \otimes x$ and $\left(\tilde{\Delta}_{M}\right)_{2}(x \otimes y)=y \otimes x \otimes y$, which implies that $\overleftarrow{d}_{\varphi}$ and $\vec{d}_{\varphi}$ vanish on such an element.

REMARK 4.6. With the Markov processes in mind, directed graphs equipped with probability vectors have always their isolated periodic orbits trivial weighted, that is, all the weights are equal to 1 on each arrow of the orbit. From now on, such orbits will always be supposed to be trivial weighted.

THEOREM 4.7. Let $\varphi$ be a Markov L-bialgebra generated by a set $\varphi_{0}$, equipped with unital coproducts $\Delta_{M}$ and $\tilde{\Delta}_{M}$. Then $\vec{d} \varphi$ and $\overleftarrow{d}_{\varphi}$ behave as Leibniz-Ito derivatives, that is, verify $\vec{d}_{\mathscr{G}}(x) \vec{d}_{\mathscr{G}}(y)=\vec{d}_{\mathscr{G}}(x y)-\vec{d}_{\mathscr{G}}(x) \delta_{R}(y)-\delta_{R}(x) \vec{d}_{\varphi}(y)$ and $\vec{d}_{\varphi}(I \otimes I)=0$. Similarly, these two equalities hold for $\overleftarrow{d}_{\varphi}$.

Proof. Let $\varphi$ be a Markov $L$-bialgebra generated by a set $\varphi_{0}$. Fix $a, b, c, d \in$ $\mathscr{G}_{0}$. Define $x:=a \otimes b$ and $y:=c \otimes d$. We get

$$
\begin{aligned}
\overleftarrow{d}_{\varphi}(x) \overleftarrow{d}_{\varphi}(y)= & \left(\left(\tilde{\Delta}_{M}\right)_{2}(x)-\delta_{L}(x)\right)\left(\left(\tilde{\Delta}_{M}\right)_{2}(y)-\delta_{L}(y)\right) \\
= & \left(\tilde{\Delta}_{M}\right)_{2}(x y)-\left(\tilde{\Delta}_{M}\right)_{2}(x) \delta_{L}(y)-\delta_{L}(x)\left(\tilde{\Delta}_{M}\right)_{2}(y) \\
& +\delta_{L}(x) \delta_{L}(y)+\left(\delta_{L}(x) \delta_{L}(y)-\delta_{L}(x) \delta_{L}(y)\right) \\
= & \overleftarrow{d}_{\mathscr{G}}(x y)-\overleftarrow{d}_{\mathscr{G}}(x) \delta_{L}(y)-\delta_{L}(x) \overleftarrow{d}_{\mathscr{G}}(y) .
\end{aligned}
$$

Similarly, for the coproduct $\vec{d}$, we can show that $\vec{d}_{\varphi}(x) \vec{d}_{\varphi}(y)=\vec{d}_{\varphi}(x y)-$ $\vec{d}_{\varphi}(x) \delta_{R}(y)-\delta_{R}(x) \vec{d}_{\varphi}(y)$. Moreover, $\vec{d}_{\varphi}(I \otimes I)=0=\overleftarrow{d}_{\varphi}(I \otimes I)$. These equations are reminiscent of those of Theorem 3.21 when $\delta_{R}$ is played by $\delta_{f}$ and $\delta_{L}$ by $\tilde{\delta}_{f}$. 
Let $\mathscr{G}$ be a finite Markov $L$-coalgebra generated, as a $k$-vector space, by an independent spanning set $\mathscr{G}_{0}$, equipped with coproducts $\Delta_{M}$ and $\tilde{\Delta}_{M}$. The present setting can be easily generalized. Fix $n>1$ and generalize the definition of $\delta_{R}$ and $\delta_{L}$ as follows: $\delta_{R, n}, \delta_{L, n}: \mathscr{G}^{\otimes n} \rightarrow \varphi^{\otimes(n+1)}$ defined by $\delta_{R, n}\left(a_{1}, \ldots, a_{n}\right)=$ $\left(a_{1}, \ldots, a_{n}\right) \otimes a_{1}$ and $\delta_{L, n}\left(a_{1}, \ldots, a_{n}\right)=a_{n} \otimes\left(a_{1}, \ldots, a_{n}\right)$, where $a_{1}, \ldots, a_{n} \in \varphi_{0}$.

THEOREM 4.8. Let $\varphi$ be a finite Markov L-coalgebra generated, as a k-vector space, by an independent spanning set $\mathscr{G}_{0}$ and equipped with coproducts $\Delta_{M}$ and $\tilde{\Delta}_{M}$. It is obtained that

(1) $\left(\delta_{L, n} \otimes \mathrm{id}\right) \delta_{R, n}=\left(\mathrm{id} \otimes \delta_{R, n}\right) \delta_{L, n}$ and $\delta_{R, n}$ and $\delta_{L, n}$ are homomorphisms;

(2) if $\vec{d}_{\varphi_{, n}}=\left(\Delta_{M}\right)_{n}-\delta_{R, n}$ and $\stackrel{\leftrightarrow}{d}_{\varphi, n}=\left(\tilde{\Delta}_{M}\right)_{n}-\delta_{L, n}$, then $\left(\overleftarrow{d}_{\varphi, n} \otimes\right.$ id $) \vec{d} \varphi_{\varphi, n}=$ $\left(\mathrm{id} \otimes \vec{d}_{\varphi, n}\right) \overleftarrow{d}_{\varphi, n}$

(3) the equality $\stackrel{\leftarrow}{\hookrightarrow}_{\varphi_{, n}}[w]=0=\vec{d} \varphi_{\varphi, n}[w]$ holds if the tensor product $[w]=$ $\left(a_{1} \otimes \cdots \otimes a_{n}\right), a_{1}, \ldots, a_{n} \in \mathscr{G}_{0}$, represents an isolated periodic orbit of period $n$ on the geometric support of the finite Markov L-coalgebra;

(4) if $\varphi$ is also a Markov L-bialgebra, the coproducts $\left(\delta_{R, n}, \delta_{L, n}\right)$ are homomorphisms. If the coproducts of $\varphi_{G}$ are unital, then $\overleftarrow{d}_{\varphi, n}$ and $\vec{d}_{\varphi, n}$ behave as Leibniz-Ito derivatives, that is, verify $\vec{d}_{\varphi, n}(x) \vec{d}_{\varphi, n}(y)=\vec{d} \vec{\varphi}_{\varphi, n}(x y)-$ $\vec{d}_{\varphi, n}(x) \delta_{R}(y)-\delta_{R}(x) \vec{d}_{\varphi_{, n}}(y)$; the same equality holds for $\overleftarrow{d}_{\varphi_{, n}}$.

Proof. The proof is straightforward.

If $C$ is a coassociative coalgebra with a group-like element $e$, then two important coproducts $\vec{d}, \overleftarrow{d}: C \rightarrow C^{\otimes 2}$ can be constructed. If $\left(\mathscr{G}, \Delta_{M}, \tilde{\Delta}_{M}\right)$ is a finite Markov $L$-coalgebra generated, as a $k$-vector space, by an independent spanning set $\mathscr{G}_{0}$, then two other important coproducts $\vec{d}_{\varphi_{, 2}}, \overleftarrow{d}_{\varphi_{, 2}}: \mathscr{G}^{\otimes 2} \rightarrow \mathscr{G}^{\otimes 3}$ can be also constructed. This remark suggests the fact that some geometric supports of coassociative coalgebras can be obtained by the line extension of some geometric supports of finite Markov L-coalgebras. Indeed, in [11], we have constructed Markov coassociative codialgebras from the line extension of geometric supports associated with a class of coassociative coalgebras.

It is interesting to notice that the role played by the coproducts $\delta_{f}$ and $\tilde{\delta}_{f}$ of the flower graph in the case of a coassociative coalgebra is played by the coproducts $\delta_{R, n}$ and $\delta_{L, n}$, creating virtual periodic orbits of period $n$ in the case of a finite Markov $L$-coalgebra of degree $n$. Observe also that

$$
\left(\overleftarrow{d}_{\varphi, n} \otimes \mathrm{id}\right) \vec{d}_{\varphi, n}[w]=0
$$

implies that the path of length $n$, represented algebraically by the tensor $[w]$, has to be a trivial-weighted isolated periodic orbit of period $n$. We sum up briefly some results in Table 4.1 .

4.1. Examples. In the following examples, from a known algebra, we construct a finite Markov $L$-coalgebra so as to the algebra turns it into a finite 
TABLE 4.1

\begin{tabular}{lll}
\hline & Coassociative coalgebra $C$ & Markov L-coalgebra $\varphi$ \\
\hline Coproducts & $\Delta_{C}$ & $\Delta \tilde{G}_{,, n}, \tilde{\Delta}_{\varphi, n}$ \\
Markovian coproducts & $\begin{array}{l}\text { g group-like, } \delta_{f}(x):=x \otimes e, \\
\tilde{\delta}_{f}(x):=e \otimes x\end{array}$ & $\delta_{R, n}, \delta_{L, n}$ \\
$(\overleftarrow{d} \otimes \mathrm{id}) \vec{d}=0$ & over primitive elements & $\begin{array}{l}\text { over-isolated orbits } \\
\text { of period } n\end{array}$ \\
$\overleftarrow{d}, \vec{d}$ & Leibniz-Ito derivative & $\begin{array}{l}\text { behave as Leibniz-Ito } \\
\text { derivative }\end{array}$ \\
\hline
\end{tabular}
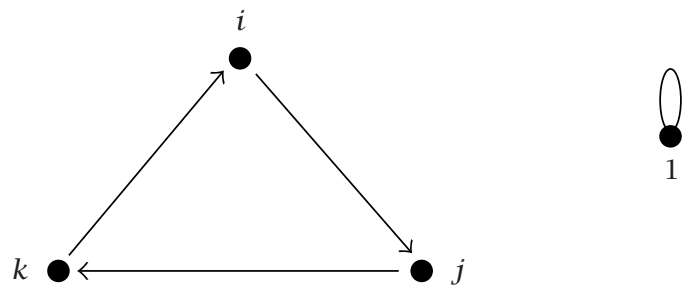

FIGURE 4.1

Markov L-Hopf algebra of degree 2. Set

$$
\mathrm{id}_{n}:=\underbrace{\mathrm{id} \otimes \mathrm{id} \otimes \cdots \otimes \mathrm{id}}_{n}, \quad n>0 .
$$

An $L$-Hopf algebra of degree $n,\left(H, \Delta_{H}, \tilde{\Delta}_{H}\right)$, is by definition an $L$-bialgebra of degree $n$, equipped with right and left counits $\tilde{\epsilon}_{H}$ and $\epsilon_{H}$ of degree $n$ such that its antipodes $S, \tilde{S}: H \rightarrow H$ verify $\left(\mathrm{id}_{n-1} \otimes m\right)\left(\mathrm{id}_{n} \otimes S\right) \Delta_{H}=\eta_{n} \epsilon_{H}$ and $(m \otimes$ id) $\left(\tilde{S} \otimes \operatorname{id}_{n}\right) \tilde{\Delta}_{H}=\tilde{\eta}_{n} \tilde{\epsilon}_{H}$, with $\eta_{n}, \tilde{\eta}_{n}: H^{\otimes(n-1)} \rightarrow H^{\otimes n}$ such that $\eta_{n}(h):=h \otimes 1_{H}$ and $\tilde{\eta}_{n}(h):=1_{H} \otimes h, h \in H^{\otimes(n-1)}$.

EXAMPLE 4.9 (the triangle graph and quaternions). Here $k=\mathbb{R}$. Recall that quaternions are defined by the associative algebra $\mathbb{H}:=\mathbb{R}\{1, i, j, k\} \backslash \mathscr{R}$, where the set of relations $\mathscr{R}$ is defined by

$$
i j=k, \quad j k=i, \quad k i=j, \quad i i=j j=k k=-1 .
$$

The quaternions fit the present formalism by considering the directed triangle graph in Figure 4.1.

Defining $x_{0} \equiv i, x_{1} \equiv j$, and $x_{2} \equiv k$ and adding subscripts $\alpha, \beta \in\{0,1,2\} \bmod 3$, that is, $x_{\alpha+\beta} \equiv x_{\alpha+\beta \bmod 3}$, we define the Markovian coproducts associated with this directed triangle as $\Delta_{M}\left(x_{\alpha}\right)=x_{\alpha} \otimes x_{\alpha+1}, \Delta_{M}(1)=\tilde{\Delta}_{M}(1)=1 \otimes 1$, and 
$\tilde{\Delta}_{M}\left(x_{\alpha}\right)=x_{\alpha-1} \otimes x_{\alpha}$. Therefore,

$$
\begin{array}{ll}
\left(\Delta_{M}\right)_{2}\left(x_{\alpha} \otimes x_{\beta}\right)=x_{\alpha} \otimes x_{\beta} \otimes x_{\beta+1}, & \epsilon_{2}\left(x_{\alpha} \otimes x_{\beta}\right)=x_{\alpha}, \\
\left(\tilde{\Delta}_{M}\right)_{2}\left(x_{\alpha} \otimes x_{\beta}\right)=x_{\alpha-1} \otimes x_{\alpha} \otimes x_{\beta}, & \tilde{\epsilon}_{2}\left(x_{\alpha} \otimes x_{\beta}\right)=x_{\beta}
\end{array}
$$

embed the directed triangle graph into a finite Markov $L$-coalgebra of degree 2.

THEOREM 4.10. (1) The algebra of quaternions embeds the triangle graph into an L-bialgebra of degree 2.

(2) Defining linear maps $S, \tilde{S}: \mathbb{U} \rightarrow \mathbb{4}$ by $S\left(x_{i}\right)=-x_{i-1}$ and $\tilde{S}\left(x_{i-1}\right)=-x_{i}$ for every $i \in\{0,1,2\}$, the L-bialgebra $\mathbb{W}$ becomes an L-Hopf algebra of degree 2 with $S$ and $\tilde{S}$ playing the role of the right and left antipodes, respectively.

(3) The linear maps $S$ and $\tilde{S}$ are unital antialgebra maps and satisfy $S \tilde{S}=$ $\mathrm{id}=\tilde{S} S$. They are the unique right and left antipodes of $\mathbb{H}$, viewed as an L-Hopf algebra of degree 2.

Proof. Let $i, i^{\prime}, j, j^{\prime} \in\{0,1,2\}$. In what follows we make computations with the right coproduct. We show that $\left(\Delta_{M}\right)_{2}$ is a unital algebra map. We get

$$
\begin{aligned}
\left(\Delta_{M}\right)_{2}\left(x_{i} \otimes x_{j}\right)\left(\Delta_{M}\right)_{2}\left(x_{i^{\prime}} \otimes x_{j^{\prime}}\right) & =\left(x_{i} \otimes x_{j} \otimes x_{j+1}\right)\left(x_{i^{\prime}} \otimes x_{j^{\prime}} \otimes x_{j^{\prime}+1}\right) \\
& =x_{i} x_{i^{\prime}} \otimes x_{j} x_{j^{\prime}} \otimes x_{j+1} x_{j^{\prime}+1}, \\
\left(\Delta_{M}\right)_{2}\left(x_{i} \otimes x_{j}\right)\left(x_{i^{\prime}} \otimes x_{j^{\prime}}\right) & =\left(\Delta_{M}\right)_{2}\left(x_{i} x_{i^{\prime}} \otimes x_{j} x_{j^{\prime}}\right) \\
& =x_{i} x_{i^{\prime}} \otimes x_{j} x_{j^{\prime}} \otimes t\left(\Delta_{M}\left(x_{j} x_{j^{\prime}}\right)\right) .
\end{aligned}
$$

Therefore, we have to prove that $t\left(\Delta_{M}\left(x_{j} x_{j^{\prime}}\right)\right)=x_{j+1} x_{j^{\prime}+1}$, which is straightforward by the following geometric proof. Suppose that $j \neq j^{\prime}$ and $\left(x_{j}, x_{j^{\prime}}\right)$ defines an edge of the triangle. This entails that $\left(x_{j+1}, x_{j^{\prime}+1}\right)$ defines the sole edge following it when we turn in a trigonometrical way. Now we observe that up to a sign the concatenation of an edge, that is, the product of its source and its terminus, gives the third vertex of the triangle. Hence, by rotation, the concatenation of $\left(x_{j+1}, x_{j^{\prime}+1}\right)$ will give the vertex just after. Therefore, up to a sign $t\left(\Delta_{M}\left(x_{j} x_{j^{\prime}}\right)\right)=x_{j+1} x_{j^{\prime}+1}$, the sign is easily obtained by noticing that if $\left(x_{j}, x_{j^{\prime}}\right)$ is an arrow of the triangle, so is $\left(x_{j+1}, x_{j^{\prime}+1}\right)$ and the sign is plus in both cases when the concatenation is realized. If the direction of $\left(x_{j}, x_{j^{\prime}}\right)$ is in the opposite sense of an existing arrow, so is $\left(x_{j+1}, x_{j^{\prime}+1}\right)$ and the concatenation will give a minus sign in both cases. In the case when $x_{j^{\prime}}$ or $x_{j}$ is the identity element, the proof is obvious since there is a loop on the identity. The case $x_{j^{\prime}}=x_{j}$ is also trivial.

The coproducts $\left(\Delta_{M}\right)_{2}$ and $\left(\tilde{\Delta}_{M}\right)_{2}$ are thus unital homomorphisms. The counits $\epsilon_{2}$ and $\tilde{\epsilon}_{2}$ are also unital algebra maps. To prove the $L$-Hopf algebra part, we must check that

$$
\begin{aligned}
& (\mathrm{id} \otimes m)(\mathrm{id} \otimes \mathrm{id} \otimes S)\left(\Delta_{M}\right)_{2}=\eta_{2} \epsilon_{2}, \\
& (m \otimes \mathrm{id})(\tilde{S} \otimes \mathrm{id} \otimes \mathrm{id})\left(\tilde{\Delta}_{M}\right)_{2}=\tilde{\eta}_{2} \tilde{\epsilon}_{2},
\end{aligned}
$$


which is straightforward with the choice we made for the right and left antipodes. The map $S$ is an antiunital map since, by definition, $-x_{i}=S\left(x_{i+1}\right)=$ $S\left(x_{i-1} x_{i}\right)$ and $S\left(x_{i}\right) S\left(x_{i-1}\right)=\left(-x_{i-1}\right)\left(-x_{i-2}\right)=\left(x_{i-1}\right)\left(x_{i-2}\right)=-\left(x_{i-2}\right)\left(x_{i-1}\right)$ $=-\left(x_{i}\right)$, so $S\left(x_{i} x_{j}\right)=S\left(x_{j}\right) S\left(x_{i}\right)$. Moreover, $S\left(x_{i} x_{j}\right)=S\left(x_{j}\right) S\left(x_{i}\right)=x_{j-1} x_{i-1}$ and $S\left(x_{i} x_{j}\right)=-S\left(x_{j} x_{i}\right)=-S\left(x_{i}\right) S\left(x_{j}\right)=-x_{i-1} x_{j-1}=x_{j-1} x_{i-1}$, proving that $S$ is well defined. The map $S$ is unital since $S(1)=S\left(x_{i}\left(-x_{i}\right)\right)=S\left(-x_{i}\right) S\left(x_{i}\right)=$ $-\left(-x_{i+1}\right)\left(-x_{i+1}\right)=1$. The map $S$ is unique since if $S_{1}$ and $S_{2}$ are two such right antipodes, we must get $x_{i} S_{1}\left(x_{i+1}\right)=x_{i} S_{2}\left(x_{i+1}\right)=1$, but $x_{i} x_{i}=-1$, so $S_{1}\left(x_{i}\right)=S_{2}\left(x_{i}\right)$. As $S_{1}$ and $S_{2}$ are equal on the generators of the quaternions, $S_{1}=S_{2}$. Moreover, $S \tilde{S}\left(x_{i}\right)=S\left(-x_{i+1}\right)=-\left(-x_{i}\right)=x_{i}$ and $\tilde{S} S\left(x_{i}\right)=\tilde{S}\left(-x_{i-1}\right)=$ $-\left(-x_{i}\right)=x_{i}$.

REMARK 4.11. The right and left antipodes $S$ and $\tilde{S}$ are not unital anticoalgebra maps.

REMARK 4.12. As a coproduct, $\Delta_{M}$ is well defined on the directed triangle graph, but is not a homomorphism for the quaternion product. If it were the case, we would get, for example, $-\Delta_{M}(k)=-\Delta_{M}(i j)=\Delta_{M}(i) \Delta_{M}(j)=-i j \otimes$ $j k=-k \otimes i$ which is true and $\Delta_{M}(-k)=\Delta_{M}(j i)=\Delta_{M}(j) \Delta_{M}(i)=j i \otimes k j=$ $(-k) \otimes(-i)$ which is still true. Yet, the $k$-linearity is lost.

EXAMPLE 4.13 (the Pauli matrices). Here $k=\mathbb{C}$. The Pauli matrices

$$
1_{2}=\left(\begin{array}{ll}
1 & 0 \\
0 & 1
\end{array}\right), \quad \gamma_{0}=\left(\begin{array}{ll}
0 & 1 \\
1 & 0
\end{array}\right), \quad \gamma_{1}=\left(\begin{array}{cc}
0 & -i \\
i & 0
\end{array}\right), \quad \gamma_{2}=\left(\begin{array}{cc}
1 & 0 \\
0 & -1
\end{array}\right)
$$

verify the relations $\gamma_{k} \gamma_{k+1}=i \gamma_{k+2}, \gamma_{k} \gamma_{k}=1_{2}$, and $\gamma_{k} \gamma_{k+1}=-\gamma_{k+1} \gamma_{k}$. Recall that $M_{2}(k)$ is the algebra generated by the Pauli matrices. This algebra fits the present formalism by considering the directed triangle graph with a loop on $1_{2}$ not represented here,
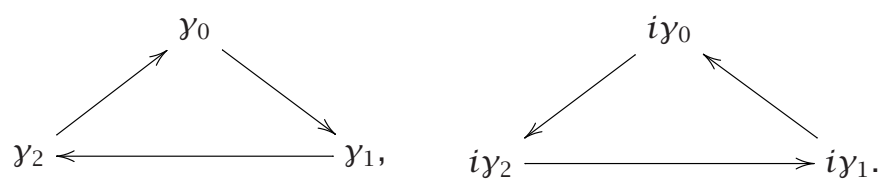

The first graph is to recall that $\gamma_{k} \gamma_{k+1}=i \gamma_{k+2}$, but it is the second one which we are interested in because it represents the relation $\left(i \gamma_{k+1}\right)\left(i \gamma_{k}\right)=\left(i \gamma_{k+2}\right)$. Defining $x_{0} \equiv i \gamma_{0}, x_{1} \equiv i \gamma_{1}$, and $x_{2} \equiv i \gamma_{2}$ and adding subscripts $\alpha, \beta \in$ $\{0,1,2\} \bmod 3$, that is, $x_{\alpha+\beta} \equiv x_{\alpha+\beta \bmod 3}$, we define $\Delta_{M}\left(x_{\alpha}\right)=x_{\alpha} \otimes x_{\alpha+1}, \Delta_{M}(1)=$ $\tilde{\Delta}_{M}(1)=1 \otimes 1$, and $\tilde{\Delta}_{M}\left(x_{\alpha}\right)=x_{\alpha-1} \otimes x_{\alpha}$. The coproducts in (4.7) embed the triangle graph into a Markov $L$-coalgebra of degree 2. 
THEOREM 4.14. (1) The algebra generated by the Pauli matrices, that is, $M_{2}(k)$, embeds the triangle graph into an L-bialgebra of degree 2.

(2) Defining linear maps $S, \tilde{S}: M_{2}(k) \rightarrow M_{2}(k)$ by $S\left(x_{i}\right)=-x_{i-1}$ and $\tilde{S}\left(x_{i-1}\right)$ $=-x_{i}$ for every $i \in\{0,1,2\}$, the L-bialgebra $M_{2}(k)$ becomes an $L$-Hopf algebra of degree 2 with $S$ and $\tilde{S}$ playing the role of right and left antipodes, respectively.

(3) The maps $S$ and $\tilde{S}$ are unital antialgebra maps and satisfy $S \tilde{S}=\mathrm{id}=\tilde{S} S$. They are the unique right and left antipodes of 뜨 viewed as an L-Hopf algebra of degree 2.

PROOF. The proof is a corollary from the quaternion example. We only stress for instance on that $S\left(x_{k}\right)=-x_{k-1}$ implies the equality $x_{k} S\left(x_{k+1}\right)=$ $-x_{k} x_{k}=-\left(i \gamma_{k}\right)\left(i \gamma_{k}\right)=\left(\gamma_{k}\right)\left(\gamma_{k}\right)=1_{2}$, which is useful for computing the antipodes equalities.

ACKNOWLedgments. The author wishes to thank Dimitri Petritis for useful discussions and fruitful advice for the redaction of this paper and R. L. Hudson as well for providing him with his results prior to their publication. He is indebted to the referees for helping him improving the readability of the text.

\section{REFERENCES}

[1] C. Cibils and M. Rosso, Hopf quivers, J. Algebra 254 (2002), no. 2, 241-251.

[2] J. Cuntz and W. Krieger, A class of $C^{*}$-algebras and topological Markov chains, Invent. Math. 56 (1980), no. 3, 251-268.

[3] R. L. Hudson, Deformed calculus and quantisation of coboundary Lie bialgebra, preprint, 2002.

[4] _ Calculus in enveloping algebras, J. London Math. Soc. (2) 65 (2002), no. 2, 361-380.

[5] S. A. Joni and G.-C. Rota, Coalgebras and bialgebras in combinatorics, Stud. Appl. Math. 61 (1979), no. 2, 93-139.

[6] A. Kumjian, D. Pask, and I. Raeburn, Cuntz-Krieger algebras of directed graphs, Pacific J. Math. 184 (1998), no. 1, 161-174.

[7] Ph. Leroux, Coassociative grammar, periodic orbits and quantum random walk over Z, preprint, 2002, http://arXiv.org/abs/quant-ph/0209100.

[8] _ From entangled codipterous coalgebras to coassociative manifolds, preprint, 2002, http://arXiv.org/abs/math.QA/0301080.

[9]__ Leibniz derivatives versus Leibniz-Ito derivatives, preprint, 2002.

[10] — On representations of braid groups determined by directed graphs, preprint, 2002, http://arXiv.org/abs/math.QA/0210260.

[11] _ , Tiling the $\left(n^{2}, 1\right)$-De-Bruijn graph with $n$ coassociative coalgebras, preprint, 2002, http://arXiv.org/abs/math.QA/0209108.

[12] J.-L. Loday, Dialgebras, Dialgebras and Related Operads, Lecture Notes in Math., vol. 1763, Springer-Verlag, Berlin, 2001, pp. 7-66.

[13] J.-L. Loday and M. Ronco, Trialgebras and families of polytopes, preprint, 2002, http://arXiv.org/abs/math.QA/0205043.

[14] S. Majid, Foundations of Quantum Group Theory, Cambridge University Press, Cambridge, 1995. 
[15] D. Quillen, Algebra cochains and cyclic cohomology, Inst. Hautes Études Sci. Publ. Math. (1988), no. 68, 139-174.

[16] K. H. Rosen, J. G. Michaels, J. L. Gross, J. W. Grossman, and D. R. Shier (eds.), Handbook of Discrete and Combinatorial Mathematics, CRC Press, Florida, 2000.

[17] M. E. Sweedler, Hopf Algebras, Mathematics Lecture Note Series, W. A. Benjamin, New York, 1969.

Philippe Leroux: Institut de Recherche en Mathématiques de Rennes, Université de Rennes 1 et UMR 6625 CNRS, Campus de Beaulieu, 35042 Rennes Cedex, France

E-mail address: phi1 ippe.1eroux@univ-rennes1.fr 


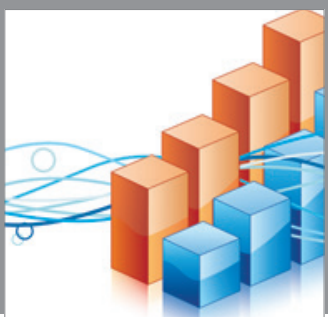

Advances in

Operations Research

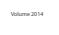

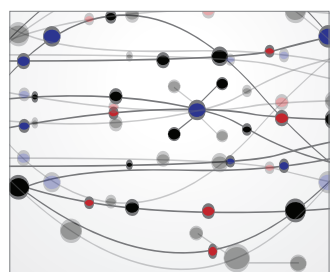

\section{The Scientific} World Journal
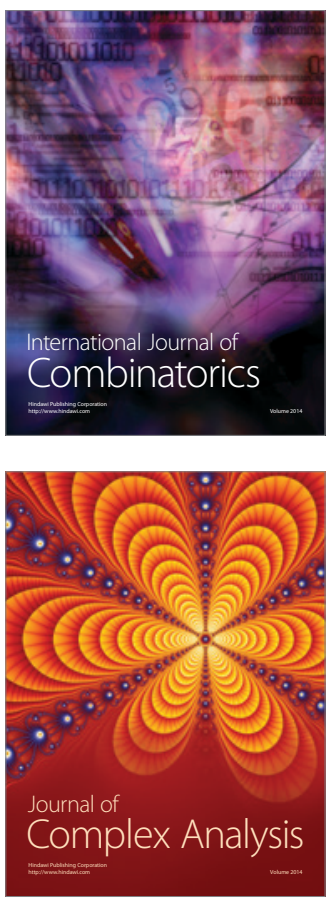

International Journal of

Mathematics and

Mathematical

Sciences
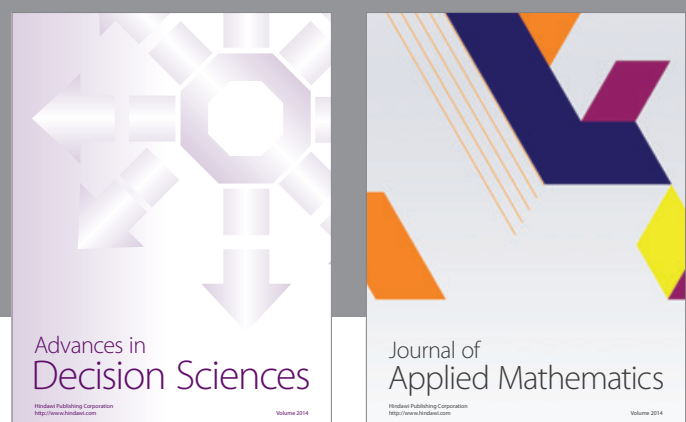

Journal of

Applied Mathematics
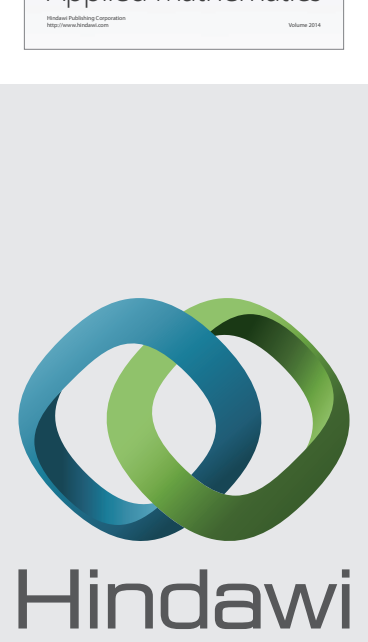

Submit your manuscripts at http://www.hindawi.com
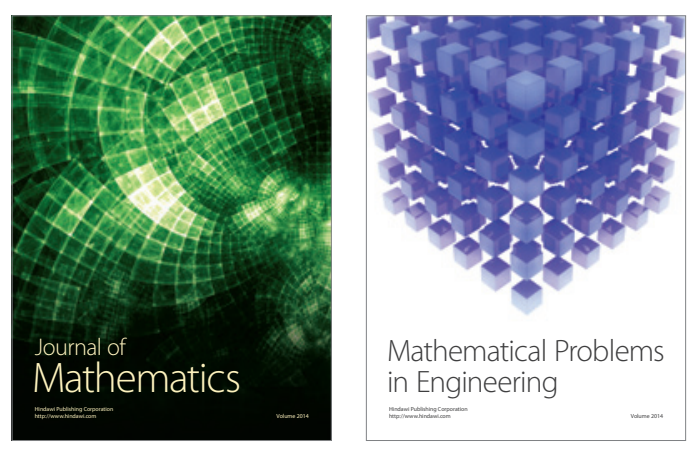

Mathematical Problems in Engineering
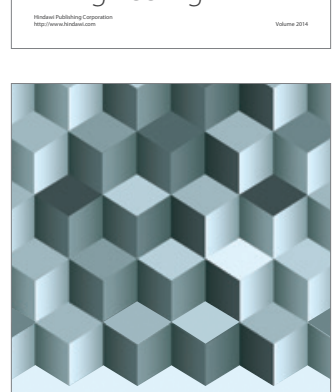

Journal of

Function Spaces
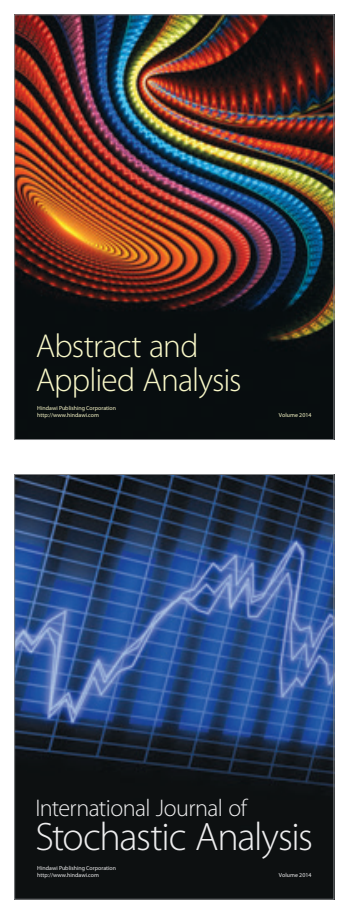

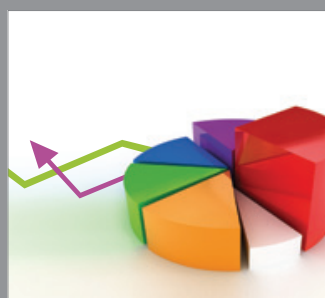

ournal of

Probability and Statistics

Promensencen
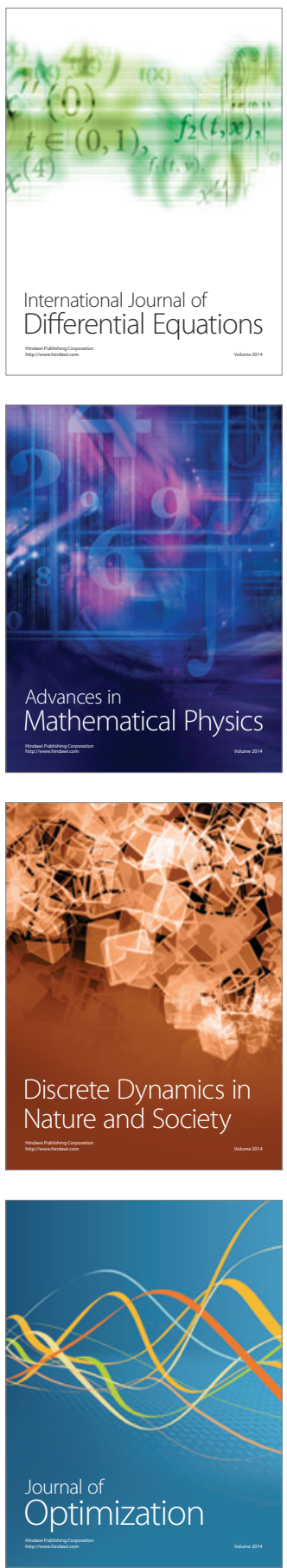